\title{
Pediatric Bilateral Blue Laser Pointer-Induced Maculopathy
}

\author{
Joseph Raevis Eric Shrier \\ Department of Ophthalmology, SUNY Downstate Medical Center, Brooklyn, NY, USA
}

\section{Keywords}

Laser pointer - Maculopathy · Retinopathy · Photic retinopathy · Solar retinopathy

\begin{abstract}
Background: We report the first case of pediatric bilateral blue laser pointer maculopathy with complete resolution of visual symptoms. Case: A 12-year-old boy presented with bilateral decreased visual acuity and central scotomata after blue laser pointer exposure. He was treated with a Medrol Dosepak and topical nonsteroidal anti-inflammatory drug (NSAID), with gradual visual acuity improved from 20/40 OU to 20/20 OU over 22 weeks, but with persistent evidence of outer retinal layer disruption from the external limiting membrane to the interdigitation zone. Conclusion: Oral steroids and topical NSAIDs may be effective in improving visual outcomes in laser pointer maculopathy in the pediatric population.
\end{abstract}

(C) 2017 The Author(s)

Published by S. Karger AG, Basel

\section{Introduction}

LASER is an acronym for "light amplification by stimulated emission of radiation." It is characterized by light with certain properties: unidirectionality, monochromaticity, and coherence. Lasers are commonly used in business and educational settings and during oral presentations as laser pointers. Laser-containing devices have become ubiquitous in our society, and their ease of availability, especially via the Internet, is cause for concern regard- 


\section{Case Reports in Ophthalmology}

ing their potential harm to vision. Most parents view all of them as novelty items and assume they are safe for their children to play with autonomously.

When certain laser pointers are directed into the eye of an individual, they are known to cause laser pointer-induced retinopathy - and if the macula is involved, laser pointerinduced maculopathy. The retinal damage from novelty lasers is mostly due to photochemical energy that cannot be dissipated quickly enough without a significant temperature elevation, leading to protein denaturation. The photochemical effect is also the cause of "snow blindness" or photokeratitis. Hemoglobin in the blood absorbs short-wavelength light $[1,2]$, and the retinal pigment epithelium is especially affected because of the high concentration of melanin, which absorbs light, especially light of shorter wavelengths. Blue lasers are particularly damaging to the macula, since light with a wavelength of $488 \mathrm{~nm}$ is best absorbed by xanthophyll, which is concentrated in the macula $[3,4]$.

Factors determining how much damage lasers can cause are broken down into dynamics inherent in the patient or the laser. Patient-related factors include the size of the pupil, the degree of retinal pigmentation, and the speed of the blink reflex. A larger pupil size can allow more light to enter, leading to increased damage. Purposeful, direct viewing would be expected to cause the greatest damage. Factors related to the laser which affect the amount of damage to the retina include frequency, duration of exposure, location of exposure, the power of the laser [5], continuous versus pulsed emission, and the angle of incidence [1].

\section{Case Report}

A 12-year-old black boy with a history of asthma presented with complaints of bilateral decreased visual acuity and central scotoma shortly after playing with a battery-powered bright blue laser pointer ( $473 \mathrm{~nm}$, according to the label on the device). While playing with the device alone at an arm's distance, he shined the blue laser pointer consecutively into both eyes, for a duration that he described as only several seconds, and immediately experienced decreased visual acuity in both eyes. He denied ever having stared at the sun and has no behavioral or psychiatric history.

The patient presented to the ophthalmology clinic 5 days after exposure and had a BCVA (best corrected visual acuity) of 20/40- OD and 20/40+3 OS. He complained of and demonstrated central scotomata on Amsler grid testing. No abnormalities were seen in the anterior segment, and the lens and vitreous were both clear in both eyes. Color fundus photographs showed bilateral yellow-orange foveal lesions, corresponding to the location of central vision (Fig. 1, 1a, 1b). A fluorescein angiogram showed early foveal hyperfluorescence with illdefined leaking bilaterally (Fig. 1, 2a, 2b). Optical coherence tomography (OCT) revealed outer retinal layer disruption from the external limiting membrane to the interdigitation zone on day 5 (Fig. 1, 3a, 3b). By week 22, the outer retinal layer disruption was more prominent (Fig. 1, 4a, 4b).

The patient was carefully observed 5 days, 2 weeks, 6 weeks, 14 weeks, and 22 weeks after exposure, with no invasive therapy performed. He was treated with a Medrol Dosepak on initial presentation, and with protracted treatment with a topical nonsteroidal antiinflammatory drug (NSAID; Ilevro) daily for 20 weeks. His BCVA gradually improved to 20/20 in both eyes by 22 weeks, and he had no residual scotoma on Amsler grid testing at that time. 


\section{Case Reports in Ophthalmology}

\section{Discussion}

We present the first case of pediatric bilateral blue laser pointer retinopathy with return to 20/20 vision in both eyes. The patient was treated with oral steroids with a Medrol Dosepak (methylprednisolone) in addition to a topical NSAID. Several reports have shown improvement in OCT findings after use of systemic steroids [6] as well as visual acuity improvement [5]. NSAIDs have been shown to improve photoreceptor survival with laser injuries in rhesus monkeys and are recommended by some retina specialists [7]. However, no randomized controlled trials exist to show definitive benefits from steroids and NSAIDs. It should be noted that steroids should be used with caution in patients with laser or solar maculopathy, since the maculopathy is known to be self-resolving, and there is a report of a patient developing central serous retinopathy after steroid treatment for solar retinopathy [8].

Shortly after solar or laser pointer exposure, patients usually present with visual acuity impairment, central and paracentral scotomas, and metamorphopsia $[1,9,10]$. While patients show gradual improvement in visual acuity [11] and in the central and paracentral scotomas, mostly with full recovery of vision over a few months, some have been left with devastating injury and permanently reduced visual acuity [12]. Our patient's central scotoma and visual acuity did not return to $20 / 20$ until 22 weeks after the exposure; however, the OCT findings still showed that significant outer retinal disruption persisted.

A major problem with laser pointers currently is that many on the market do not meet the federal guidelines for safety, specifically when bought from overseas via the Internet. The US Food and Drug Administration (FDA) has set a limit of $5 \mathrm{~mW}$ for the power output of laser pointers in the visible wavelength range $(400-710 \mathrm{~nm})$ and a limit of $2 \mathrm{~mW}$ in the infrared portion of the light spectrum [13]. However, researchers at the National Institute of Standards and Technology looked at 122 commercially bought laser pointers and showed that $90 \%$ of the green laser pointers did not meet the FDA guidelines. In addition, half of these devices were emitting power of at least twice the federal limit, and $75 \%$ of the green laser pointers were emitting infrared light in excess of the 2-mW limit [14].

With 1,000- to 2,000-mW blue and violet laser pointers now being easily accessible via the Internet, the rise in permanent visual impairment from laser pointers will likely continue in the near future. Over a 1-year span during 2014 in Saudi Arabia, researchers reported 14 cases of blue laser pointer injuries to the retina including full-thickness macular holes and prehyaloid hemorrhages with epiretinal membrane formation [15].

Laser pointers that emit longer wavelengths of light, such as red, theoretically cause less harm and are potentially safer than those emitting shorter wavelengths, such as green [4] and, especially, blue [14], both of which pose a greater threat to vision. However, determining differences between powers of laser pointers can be difficult for consumers, and even if the labels are read correctly, they frequently are inaccurate [14]. This puts the pediatric population at risk from misuse of laser pointers. Children and their parents would certainly benefit from increased awareness and improved labeling.

\section{Acknowledgements}

We would like to thank Research to Prevent Blindness for an unrestricted departmental grant. 


\section{Statement of Ethics}

The authors of this study declare no ethical conflicts.

\section{Disclosure Statement}

The authors declare that they have no conflicts of interest

\section{References}

1 Yiu G, Itty S, Toth C: Ocular safety of recreational lasers. JAMA Ophthalmol 2014;132:245-246

-2 Lund DJ, Peter EP, Stuck BE: Ocular hazards of Q-switched blue wavelength lasers. Spie Proc 2001;4246. DOI: $10.1117 / 12.426722$.

-3 King A, Gottlieb E, Brooks DG, Murphy MP, Dunaief JL: Mitochondria-derived reactive oxygen species mediate blue light-induced death of retinal pigment epithelial cells. Photochem Photobiol 2004;79:470-475.

4 Robertson DM, McLaren JW, Salomao DR, Link TP: Retinopathy from a green laser pointer: a clinicopathologic study. Arch Ophthalmol 2005;123:629-633.

Barkana Y, Belkin M: Laser eye injuries. Surv Ophthalmol 2000;44:459-478.

Hossein M, Bonyadi J, Soheilian R, Soheilian M, Peyman GA: SD-OCT features of laser pointer maculopathy before and after systemic corticosteroid therapy. Ophthalmic Surg Lasers Imaging 2011;42 online:e135-e138.

-7 Brown J Jr, Hacker H, Schuschereba ST, Zwick H, Lund DJ, Stuck BE: Steroidal and nonsteroidal antiinflammatory medications can improve photoreceptor survival after laser retinal photocoagulation. Ophthalmology 2007;114:1876-1883.

8 Bouzas EA, Moret P, Pournaras CJ: Central serous chorioretinopathy complicating solar retinopathy treated with glucocorticoids. Graefes Arch Clin Exp Ophthalmol 1999;237:166-168.

-9 Rusu I, Sherman J, Gallego-Pinazo R, Lam M, Freund KB: Spectral-domain optical coherence tomography and fundus autofluorescence findings in a case of laser pointer-induced maculopathy. Retin Cases Brief Rep 2013;7:371-375.

10 Vukicevic M, Gin T, Kell S: Laser pointer retinal injury: a case report. Aust Orthopt J 2014;46:14-16.

-11 Ueda T, Kurihara I, Koide R: A case of retinal light damage by green laser pointer (Class 3b). Jpn J Ophthalmol 2011;55:428-430.

12 Wyrsch S, Baenninger PB, Schmid MK: Retinal injuries from a handheld laser pointer. N Engl J Med 2010;363:1089-1091.

13 US Food and Drug Administration: Important information for laser pointer manufacturers. 2015. http://www.fda.gov/Radiation-

EmittingProducts/RadiationEmittingProductsandProcedures/HomeBusinessandEntertainment/Laser ProductsandInstruments/ucm116373.htm (accessed January 2016).

14 National Institute of Standards and Technology: NIST tests underscore potential hazards of green laser pointers. 2013. http://www.nist.gov/pml/div686/pointer-032013.cfm.

15 Alsulaiman SM, Alrushood AA, Almasaud J, et al: High-power handheld blue laser-induced maculopathy: the results of the King Khaled Eye Specialist Hospital Collaborative Retina Study Group. Ophthalmology 2014;121:566-572. 


\section{Case Reports in Ophthalmology}
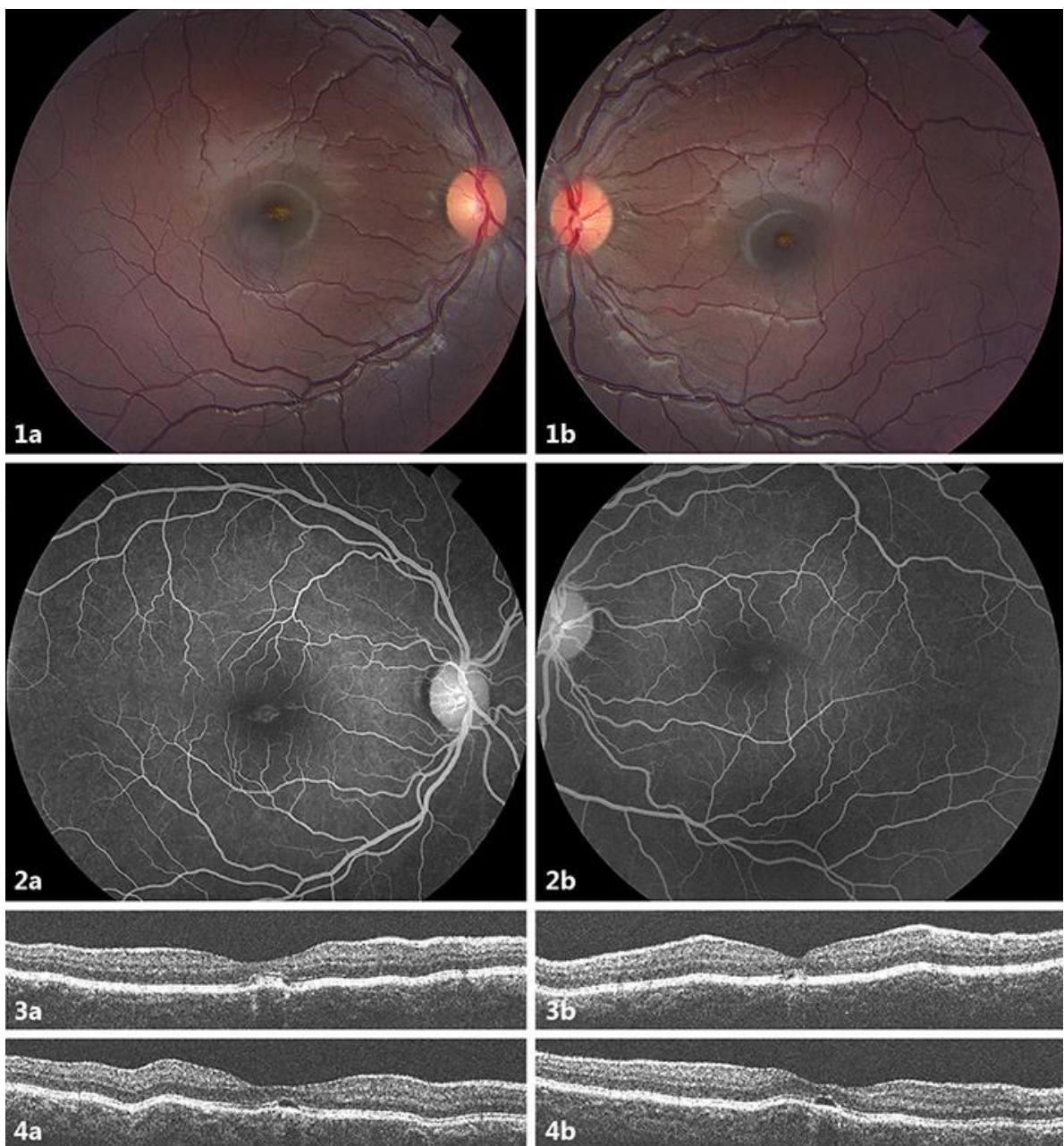

Fig. 1. Color fundus photographs taken 5 days after laser pointer injury. 1a, $\mathbf{1 b}$ The images show bilateral yellow-orange foveal lesions. These changes are reminiscent of solar retinopathy. 2a, $\mathbf{2 b}$ Fluorescein angiogram fundus photographs showing early foveal hyperfluorescence with ill-defined macular leaking bilaterally. 3a, 3b, 4a, 4b Optical coherence tomography images 5 days (3a, 3b) and 22 weeks $(\mathbf{4 a}, \mathbf{4 b})$ after the inciting laser pointer injury. Outer retinal layer disruption from the external limiting membrane to the interdigitation zone is present on day 5 , and the outer retinal disruption becomes more evident by week 22. 